\title{
sciendo
}

Int. J. of Applied Mechanics and Engineering, 2021, vol.26, No.3, pp.177-186

DOI: 10.2478/ijame-2021-0043

\section{AN EFFECT OF ELECTROKINETICS PHENOMENA ON NONLINEAR WAVE PROPAGATION IN BUBBLY LIQUIDS}

\author{
G. PANAHOV and E. ABBASOV* \\ Department of Fluid Mechanics, Institute of Mathematic and Mechanics \\ Azerbaijan National Academy of Sciences, B. Vagabzadeh St., 9, AZ1141, Baku, AZERBAIJAN \\ E-mail: eldar.abbasov@imm.az \\ S. BAKHTIYAROV \\ New Mexico Institute of Mining and Technology, Mechanical Engineering Department \\ 801 Leroy Place, Socorro, NM 87801, USA \\ P. MUSEIBLI \\ Department of Fluid Mechanics, Institute of Mathematic and Mechanics \\ Azerbaijan National Academy of Sciences, B. Vagabzadeh St., 9, AZ1141, Baku, AZERBAIJAN
}

\begin{abstract}
A study of nonlinear waves in liquid-gas mixtures with the consideration of internal effects is an important problem of both the fundamental and the applied fluid mechanics. Investigation of nonlinear waves in the gasliquid mixtures with allowance for internal effects is an important task of both fundamental and applied fluid mechanics. These problems often arise in industrial processes such as oil and gas production, hydrocarbons pipeline transportation, gas-saturated fluids flow in pipelines, etc. In this work, we investigate the effect of the internal electric field on the nonlinear wave propagation in a bubbly liquid. Numerical simulations have been conducted to study the nonlinear waves described by the nonlinear Burgers-Korteweg-de Vries equation. The numerical simulations showed that the electrokinetic processes significantly affect the wave propagation process. The amplitude of the waves gradually decreases when the size of the gas bubble is decreasing and the electrical potential increases. A good agreement of obtained results with our previous predictions is found.
\end{abstract}

Keywords: nonlinear waves, bubbly liquids, electrokinetic phenomena, perturbation, electric potential

\section{Introduction}

A study of nonlinear waves in liquid-gas mixtures with the consideration of internal effects is an important problem of the fluid mechanics. There are many engineering and industry applications related to the liquid flow with gas bubbles. An extensive literature review of both theoretical and experimental studies of the various aspects of propagation of pressure waves of small amplitude in bubbly liquids has been done by Wijngaarden [1]. The previous studies showed that the dynamic properties of bubbly liquids could be beneficially used in the design of systems to drive high-speed vessels. In this review paper, the authors Feng and Meng and Wang et al. [2,3] discussed the effects of nonlinearity, dispersion and dissipation described by Burgers' and Korteweg and de Vries equations.

Macroscopic equations for sound propagation in a bubbly liquid at finite volume fraction in the linearized, low-frequency regime are derived by Caflisch et al. [4]. For the small volume fraction, the results can describe the relative drift and deformations of the bubbles. At finite volume fractions the incompressibility of the gas is a consequence of conservation of local liquid volume due to the low frequencies. It is shown that

\footnotetext{
${ }^{*}$ To whom correspondence should be addressed
} 
the compressibility effects are exposed for the microscopic problem at second order and the leading-order macroscopic equations are affected by the compressibility.

The propagation of nonlinear waves in a mixture of liquid and gas bubbles considering the effect of heat transfer and viscosity was studied by Kudryashov and Sinelshchikov [5]. The authors applied different scales of time and coordinate and the method of perturbation to obtain nonlinear evolution equations for describing pressure waves. A nonlinear evolution equation of the fourth order was derived for describing the pressure waves in a liquid with bubbles with consideration of the effect of the viscosity and the heat transfer on the pressure waves. The obtained nonlinear differential equation generalizes the Burgers equation, the Korteweg-de Vries equation, the Korteweg-de Vries-Burgers equation and the Kuramoto-Sivashinsky equation. The authors found some exact solutions of the derived non-integrable equation.

Long weakly non-linear waves in the liquid containing gas bubbles were studied by Kudryashov and Sinelshchikov [5]. The authors derived equations for non-linear waves taking into consideration high order terms in the asymptotic expansion. The derived equations describe non-linear waves at different length and time scales and are the generalizations of the Burgers and the Burgers-Korteweg-de Vries equations. The authors suggested that the equations derived for the non-linear waves in the liquid containing gas bubbles can be used for the description of non-linear waves in other media (viscoelastic tubes, plasma) as well.

An explicit form of a wave equation in a bubbly liquid medium is derived by Mahmood and Kwak [6]. A dispersion relation for the linear pressure wave propagation in bubbly liquids was obtained using the linearized wave equation and the Keller-Miksis equation by Keller and Miksis [7] for bubble wall motion. It is shown that the attenuation of the waves in a bubbly liquid takes place due to the viscosity and the heat transfer from/to the bubble. The thermal diffusion has a considerable effect on the frequency dependent attenuation coefficients at the lower frequency region as reported by Yuning et al. [8]. The authors demonstrated that the phase velocity and the attenuation coefficient obtained from the dispersion relation are in a good agreement with the observed values in all sound frequency ranges from $\mathrm{kHz}$ to $\mathrm{MHz}$.

An analytical approach for the shock propagation in a bubbly mixture is provided by Seung and Kwak [9]. An explicit form of the wave equation in a bubbly mixture was obtained in this study. The solution of the derived wave equation provides the pressure field due to the shock profile and the radiation pressure field considering the interaction between bubbles. The equation of motion of the bubble along the shock wave which shows the relaxation oscillation was obtained by using the Keller-Miksis equation. A rigorous treatment for the heat transfer from/to the bubble was considered based on the homologous solutions for the mass, momentum and energy equations for the gas inside the bubble by Martins and Seleghim [10]. The heat transfer at the interface was taken into account by obtaining the thickness of the thermal boundary layer from the heat diffusion equation for the liquid layer outside the bubble wall by Gubaidullin and Fedorov [11]. An analytical study for the relaxation oscillation provides an understanding of the relaxation oscillations in the shock front in a bubbly mixture and the interaction between the bubbles. It is also demonstrated that heat transfer through the bubble wall significantly affects the amplitude of the relaxation oscillations. However, the bubble-bubble interaction affects mainly the period of the oscillations occurring behind the shock front.

In previous studies of nonlinear waves in the liquid containing gas bubbles only a surface tension, a liquid viscosity and an interphase heat transfer were taken into account by Li et al. and Yano et al. $[12,13]$. However, during the flow of the bubbly liquid, some other important physical phenomena such as an interaction between dissipative and dispersive processes in a gas-liquid mixture and its effect on the wave propagation may take place. Therefore, a study of nonlinear waves in liquid-gas mixtures with consideration of the internal physical effects is an important problem of the fluid mechanics and was made by Sitdikova and Gimaltdinov [14]. In this study we have investigated the nonlinear waves in a liquid phase with gas bubbles taking into account an effect of the internal electric field (Cerepi [15]). To the best of our knowledge, an effect of the potential difference on the nonlinear wave propagation was not studied previously. In this study the effect of the electrokinetic processes on the propagation of nonlinear waves was analyzed using a basic system of equations for the nonlinear waves and the obtained equation was solved numerically. Also, an experimental study was conducted to investigate the effect of the electrokinetic processes on the fluid flow characteristics. 


\section{Experimental setup and procedures}

The experimental studies were conducted to study the impact of the flow rate on the electric potential differences generated during electrokinetic processes in the fluid flow. The effect of the potential difference on the formation of gas bubbles and fluid dynamics was previously investigated by Panahov and Museibli [16]. In these experiments we studied the flow of an aqueous solution of sodium chloride in a circular pipe by measuring the flow rate, the potential difference and the electrical conductivity of the test solutions at different electrolyte concentrations. The experimental setup used for these purposes is schematically shown in Fig.1. The experimental setup consists of a compressed gas cylinder (1), valves $(2,5)$, a pressure gauge (3), a container for the gas-liquid mixture system (4), a potentiometer to record the electrokinetic potential (6), $2.7 \mathrm{~m}$ long and $4 \mathrm{~mm}$ diameter circular pipe (7), an ultra-thermostat (8), and a graduated cylinder (9).

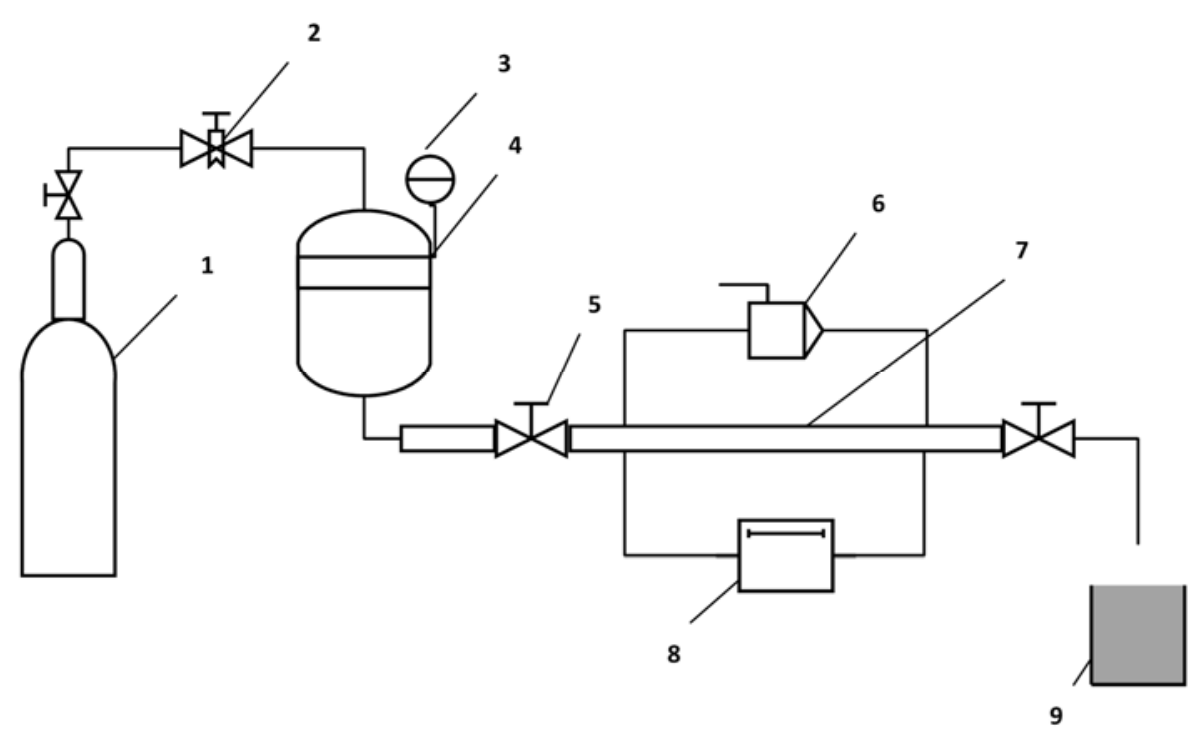

Fig.1. Experimental setup.

The experiments were conducted using tap water with and without the electrolyte (sodium chloride $\mathrm{NaCl}$ ) additive. The mineral content in the water (without the electrolyte additive) is shown in Tab.1. The sodium chloride $(\mathrm{NaCl})$ dissolved in water disassociates into cations $\left(\mathrm{Na}^{+}\right)$and anions $\left(\mathrm{Cl}^{-}\right)$. In the first set of the experiments, we studied a variation of the electric potential difference of the water on the volume flow rate. The results of the experiments are shown in Fig.2.

Table 1. The mineral content in the water.

\begin{tabular}{|c|c|c|c|c|c|c|c|c|c|}
\hline $\mathrm{Ca}^{2+}$ & $\mathrm{Mg}^{2+}$ & $\mathrm{Ca}^{2+}+\mathrm{Mg}^{2+}$ & $\mathrm{Na}^{+}$ & $\mathrm{Cl}^{-}$ & $\mathrm{SO}_{4}^{2-}$ & $\mathrm{HO}_{3}^{-}$ & $\Sigma K$ & $\mathrm{SiO}_{3}^{2-}$ & Salinity \\
\hline \multicolumn{10}{|c|}{$m g / l$} \\
\hline 3.8 & 2.2 & 6.0 & 0.5 & 0.2 & 1.9 & 4.4 & 6.5 & 18.2 & 345 \\
\hline
\end{tabular}

A variation of the electrical conductivity in the test solution at various concentrations of the sodium chloride was studied experimentally using a conductometer. Figure 3 shows a variation of the electrical conductivity of the test solutions with electrolyte $(\mathrm{NaCl})$ concentration. As seen from this figure, the electrical conductivity of the solution almost linearly increases with the electrolyte concentration. 


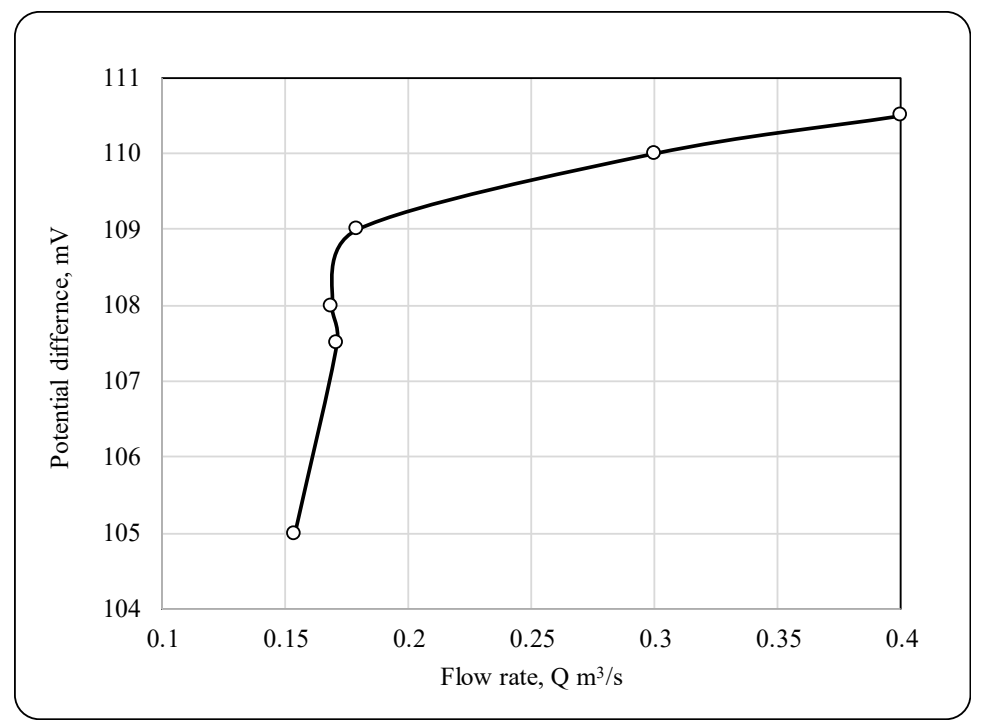

Fig.2. The variation of potential difference vs. volume flow rate of water.

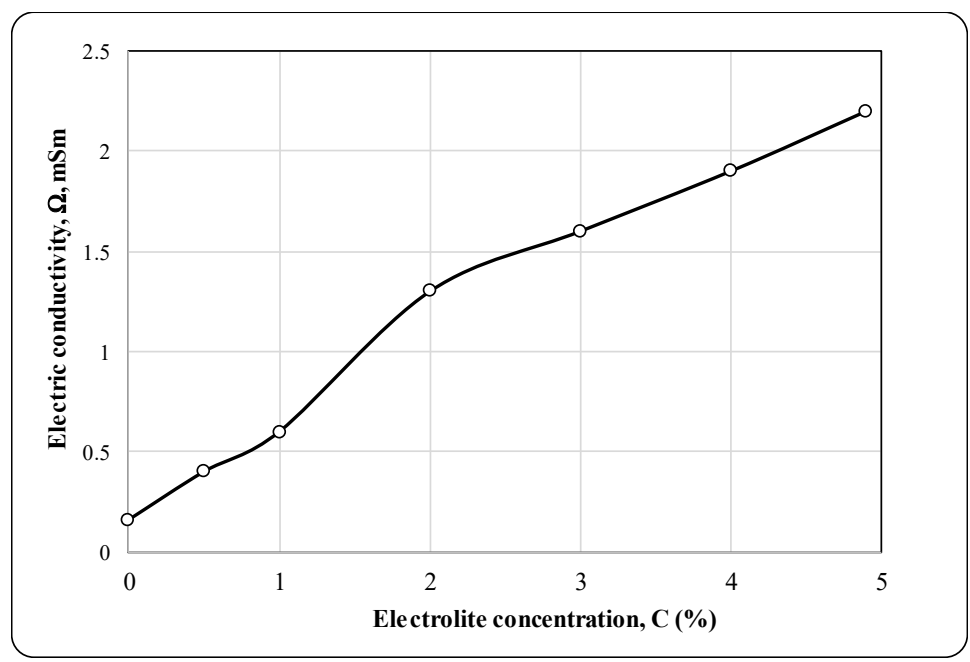

Fig.3. The electrical conductivity vs. $\mathrm{NaCl}$ concentration.

\section{Mathematical modeling of nonlinear wave propagation in bubbly liquid}

Nonlinear effects arising during the electrolyte solutions flow are also encountered in pipeline transportations of oil and it is essentially difficult to control these processes. It is important to note that the high-frequency wave processes in some cases reduce the efficiency of the transportation process. However, in some cases the high-frequency oscillations can be a control factor for the restoration of the pipeline's throughput (for example, a destruction of the gas accumulations, prevention of the sedimentation of solid phase, etc.). Therefore, it is important to determine the origins of such processes, the ranges of possible frequencies and amplitudes of the oscillations, and selection of the appropriate device (technique) for recording of these high-frequency waves. Previous studies (Gallyamov et al. [17]) show that in pipeline transportation the oil has a gas content of up to $2 \%$. Transport of such media is accompanied by highfrequency oscillations, which cause early development of cavitation, enhanced corrosion processes, etc.

The problem of two-phase flows is traditionally solved by using a system of differential equations describing both liquid and gas phases. However, assuming that in this case the gas content is insignificant, 
a single-phase model developed by Nigmatulin [18] has been used in our mathematical simulations. It is assumed that the characteristic linear dimension of the perturbation is much greater than the distance between the bubbles and, in addition, the distance between the bubbles is different from the radius of the bubble. Under these assumptions the motion of such a system can be considered using a homogeneous fluid approach proposed by Nakoryakov et al. [19]. According to this approach, the entire mixture is a continuous medium of density equal to the density of the liquid, and the compressibility is equal to the compressibility of the gas. With these assumptions, the density of the mixture can be expressed as:

$$
\rho=\rho_{1}^{0} a_{1}+\rho_{2}^{0} a_{2} \approx \rho_{1}^{0} a_{1} .
$$

The change of the density can be expressed as:

$$
\delta \rho=-\rho_{1}^{0} \delta a_{2}
$$

where the volume concentration of the gas phase:

$$
a_{2}=\frac{4}{3} \pi R^{3} n
$$

The continuity equations for the number of the bubbles per unit of volume of the gas-liquid mixture $n$ and the density $\rho$ can be written as:

$$
\frac{\partial n}{\partial t}+\frac{\partial(n U)}{\partial x}=0, \quad \frac{\partial \rho}{\partial t}+\frac{\partial(\rho U)}{\partial x}=0
$$

where $U$ is the velocity of the homogeneous medium. From the last two equations we can obtain:

$$
\frac{\partial n}{n}=\frac{\partial \rho}{\rho}
$$

Combining Eqs (3.2) and (3.3) we can obtain:

$$
\delta a_{2}=\frac{4}{3} \pi\left(3 R^{2} n \delta R+R^{3} \delta n\right)=3 \frac{a_{2}}{R} \delta R+\frac{a_{2}}{\rho} \delta \rho .
$$

Substituting the equation (3.4) into the equation (3.1) we can obtain:

$$
\delta \rho=-\rho_{1}^{0}\left(\frac{3 a_{2}}{R}+\frac{a_{2}}{\rho} \delta \rho\right)
$$

or

$$
\delta \rho=-\frac{3 a_{1} a_{2} \rho_{1}^{0}}{R} \delta R
$$

The radial motion of the gas bubble in an infinite incompressible fluid (excluding phase transitions and surface tension) can be described by the Rayleigh-Lamb equation (Kutateladze and Styrikovich [20]): 


$$
R \frac{d^{2} R}{d t^{2}}+\frac{3}{2}\left(\frac{d R}{d t}\right)^{2}=\frac{1}{\rho_{1}^{0}}\left(P_{2}-P_{1}-\frac{4 \mu}{R} \frac{d R}{d t}-\frac{2}{3} \sigma E R \frac{d R}{d t}\right)
$$

Assuming that the gas inside the bubble is in the barotropic condition: $P_{2}=P_{0}\left(\rho_{2}^{0} / \rho_{20}^{0}\right)^{\gamma}$, then for the long waves the isothermal approximation $(\gamma=1)$ will be valid.

For short waves a heat exchange between the gas and the liquid phases can be neglected, and the gas phase can be considered in adiabatic condition. However, the basic assumptions of the homogeneous model will be violated if the phase velocity does not have time to equalize. Since there are no phase transitions, the mass of a single bubble will be conserved:

$$
P_{2}=P_{0}\left(R_{0} / R\right)^{3 \gamma}
$$

Then Eq.(3.6) can be written as:

$$
P_{l}=P_{0}\left(R_{0} / R\right)^{3 \gamma}-\left(\frac{4 \mu}{R_{0}}+\frac{2}{3} \sigma E R_{0}\right) \frac{d R}{d t}-\rho_{1}^{0} R \frac{d^{2} R}{d t^{2}}-\frac{3}{2} \rho_{1}^{0}\left(\frac{d R}{d t}\right)^{2} .
$$

From this equation we can find a pressure increment [20]:

$$
\delta P=-\frac{3 \gamma P_{0}}{R_{0}} \delta R-\left(\frac{4 \mu}{R_{0}}+\frac{2}{3} \sigma E R_{0}\right) \frac{d(\delta R)}{d t}-\rho_{1}^{0} R_{0} \frac{d^{2}(\delta R)}{d t^{2}} .
$$

From Eq.(3.5) we can obtain the following equation of state:

$$
\delta P=\frac{\gamma P_{0}}{a_{1} a_{2} \rho_{1}^{0}} \delta \rho+\frac{\left(\frac{4 \mu}{R_{0}}+\frac{2}{3} \sigma E R_{0}\right) R_{0}}{3 a_{1} a_{2} \rho_{1}^{0}} \frac{d(\delta \rho)}{d t}+\frac{R_{0}^{2}}{3 a_{1} a_{2}} \frac{d^{2}(\delta \rho)}{d t^{2}}
$$

where

$$
C_{e}^{2}=\frac{\gamma P_{0}}{a_{1} a_{2} \rho_{1}^{0}}
$$

is the equilibrium velocity of the sound propagation in the mixture (Nigmatulin [18]).

The continuity and the momentum equations for the homogeneous gas-liquid medium can be written as:

$$
\begin{aligned}
& \frac{\partial \rho}{\partial t}+\frac{\partial \rho U}{\partial x}=0, \\
& \rho \frac{\partial U}{\partial t}+\rho U \frac{\partial U}{\partial x}+\frac{\partial P}{\partial x}=0 .
\end{aligned}
$$

Equations (3.7)-(3.9) are one-dimensional Navier-Stokes-Boussinesq equations system. If the coefficients of $\frac{d(\delta \rho)}{d t}$ and $\frac{d^{2}(\delta \rho)}{d t^{2}}$ are small, the time derivatives can be approximately expressed as: 


$$
\frac{d(\delta \rho)}{d t}=-\rho \frac{\partial U}{\partial x}, \quad \frac{d^{2}(\delta \rho)}{d t^{2}}=C_{e}^{2} \frac{\partial^{2} \rho}{\partial x^{2}}
$$

Substituting Eq.(3.7) into Eqs (3.8) and (3.9) we can write:

$$
\begin{aligned}
& \frac{\partial U}{\partial t}+U \frac{\partial U}{\partial x}+\frac{C_{e}^{2}}{\rho} \frac{\partial \rho}{\partial x}=2 \eta \frac{\partial^{2} U}{\partial x^{2}}-\frac{2 \beta C_{e}}{\rho} \frac{\partial^{2} U}{\partial x^{3}}, \\
& \frac{\partial \rho}{\partial t}+\rho \frac{\partial U}{\partial x}+U \frac{\partial \rho}{\partial x}=0
\end{aligned}
$$

where

$$
2 \eta=\frac{\left(\frac{4 \mu}{R_{0}}+\frac{2}{3} \sigma E R_{0}\right)}{3 a_{1} a_{2} \rho_{1}^{0}}, \quad 2 \beta=\frac{R_{0} C_{e}}{3 a_{1} a_{2}} .
$$

If $\eta=\beta=0$, the system of equations (3.10)-(3.11) describes the "simple waves" or Riemann waves. But in general, the system of equations (3.10)-(3.11) does not contain solutions of the Riemann type. In case of the weak dispersion and dissipation, the solutions of the system can be sought in the form of a quasi-simple wave as in Karpman [21] and Whitham [22]:

$$
\delta \rho=f(U)+\psi(x, t),
$$

where $f(U)$ is the solution of the system (3.10) and (3.11) without dispersion and dissipation, $\psi(x, t)$ is a function, depending on dissipation and dispersion effects. If $\eta=\beta=\psi=0$, we have:

$$
d f(U) / d U= \pm \rho / C
$$

If we consider the perturbations propagating along the characteristics $x-C_{e} t=$ Const, then it will correspond to the positive sign on the right-hand side of Eq.(3.13). Combining Eqs (3.10)-(3.13) we will obtain the following expression:

$$
-\frac{C_{e}^{2}}{\rho} \frac{\partial \psi}{\partial x}+\frac{C_{e}}{\rho} \frac{\partial \psi}{\partial t}+2 \eta \frac{\partial^{2} U}{\partial x^{2}}-2 \beta C_{e} \frac{\partial^{3} U}{\partial x^{3}}=0
$$

Equation (3.14) can be easily integrated along the characteristics $x-C_{e} t=$ Const by introducing the variable $x-C_{e} t=z$ :

$$
\psi=\frac{\rho}{C_{e}^{2}}\left(\eta \frac{\partial U}{\partial z}-\beta \frac{\partial^{3} U}{\partial z^{3}}\right) .
$$

Substituting this expression into Eqs (3.10) or (3.11), we will obtain an equation for the velocity perturbations in the coordinate system moving with the velocity $C_{e}$, which is the classical Burgers-Korteweg-de-Vries equation: 


$$
\frac{\partial U}{\partial t}+U \frac{\partial U}{\partial z}-\eta \frac{\partial^{2} U}{\partial z^{2}}+\beta \frac{\partial^{2} U}{\partial z_{2}}=0
$$

where

$$
\eta=\frac{\left(\frac{4 \mu}{R_{0}}+\frac{2}{3} \sigma E R_{0}\right) R_{0}}{6 a_{1} a_{2} \rho_{1}^{0}}, \quad \beta=\frac{R_{0}^{2} C_{e}}{6 a_{1} a_{2}} .
$$

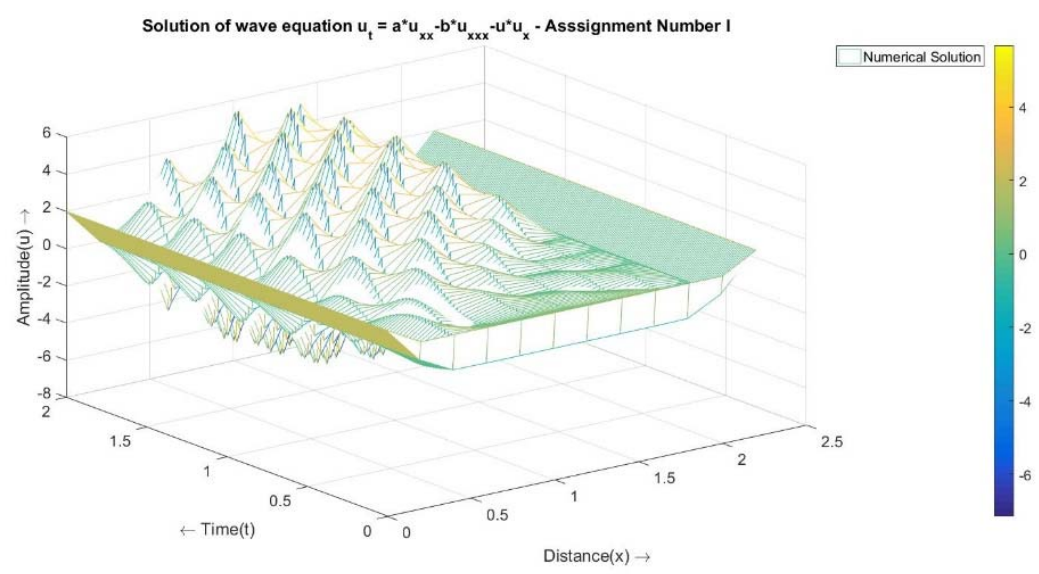

Fig.4. The patterns of nonlinear waves propagation at $R_{0}=0.1 \mathrm{~mm}$ and $E=2 \mathrm{mV}$.

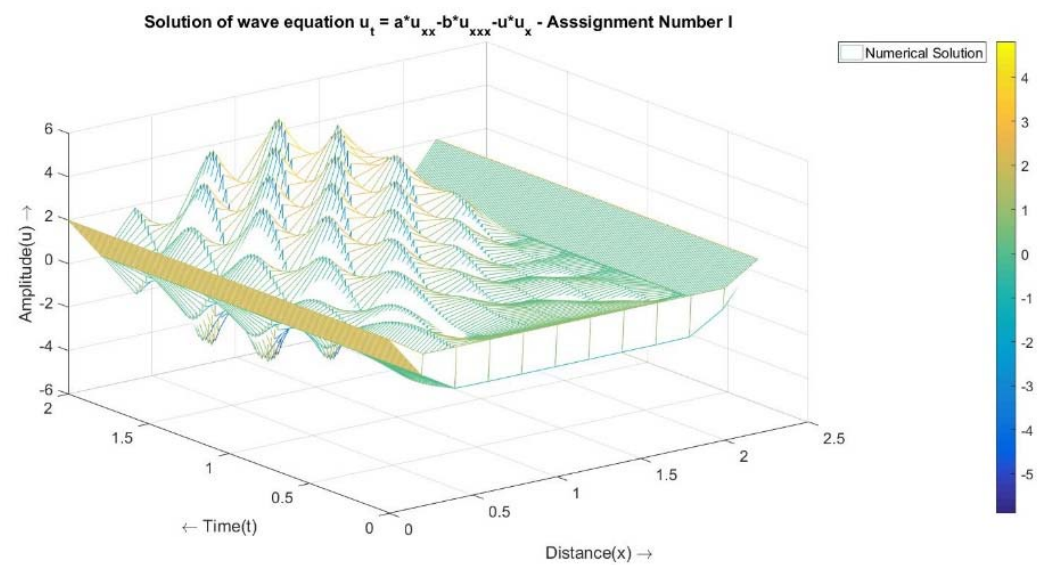

Fig.5. The patterns of nonlinear waves propagation at $R_{0}=0.08 \mathrm{~mm}$ and $E=3 \mathrm{mV}$.

Equation (3.15) for the nonlinear wave processes has been solved numerically. The patterns of the nonlinear wave propagation for various $R_{0}(0.1 \mathrm{~mm}, 0.08 \mathrm{~mm}$ and $0.06 \mathrm{~mm})$ and $E(2 \mathrm{mV}, 3 \mathrm{mV}$ and $5 \mathrm{mV})$ are shown in Figs 4-6. As seen from these figures, the amplitude of the waves gradually decreases when the size of the gas bubble is decreasing and the electrical potential increases. In other words, the electrokinetic processes significantly affect the wave propagation process. This is in a good agreement with our previous studies which showed that the potential difference increases as the radii of the bubble decrease, and the waves attenuate gradually (Panahov and Museibli [16]). 


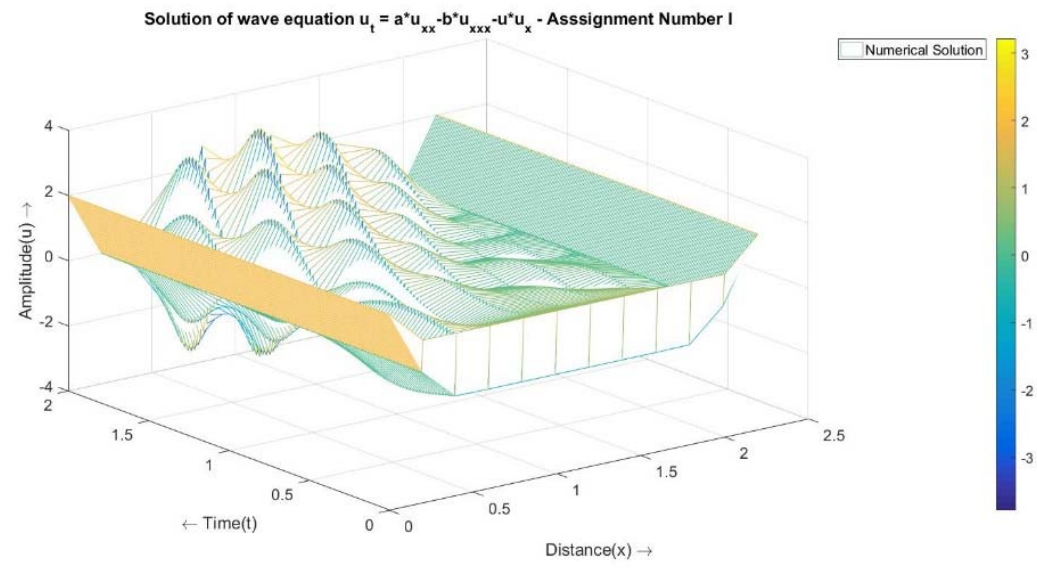

Fig.6. The patterns of nonlinear waves propagation at $R_{0}=0.06 \mathrm{~mm}$ and $E=5 \mathrm{mV}$.

\section{Conclusions}

A propagation of the long nonlinear waves in a liquid with gas bubbles has been studied with the consideration of the elektrokinetic process, which occurs during the fluid flow. The nonlinear differential equation (Burgers-Korteweg-de-Vries) with respect to the potentials difference parameter for the long weak nonlinear waves in the bubbly liquid has been derived. The numerical simulations showed that the electrokinetic processes significantly affect the wave propagation. The amplitude of the waves gradually decreases when the size of the gas bubble is decreasing and the electrical potential increases. A good agreement of obtained results with our previous predictions is found.

\section{Nomenclature}

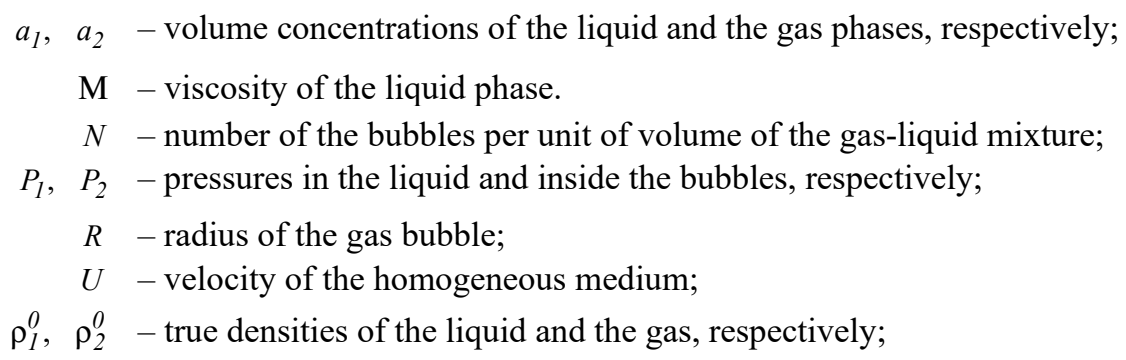

\section{Acknowledgements}

This research did not receive any specific grant from funding agencies in the public, commercial, or not-for-profit sectors.

\section{References}

[1] Wijngaarden L.V. (1972): One-dimensional flow of liquids containing small gas bubbles.- Annual Review of Fluid Mechanics, vol.4, No.1, pp.369-396. https://doi.org/10.1146/annurev.f1.04.010172.002101

[2] Feng Zs. and Meng Qg. (2007): Burgers-Korteweg-de Vries equation and its traveling solitary waves.- Sci. China Ser. A., vol.50, pp.412-422. https://doi.org/10.1007/s11425-007-0007-6 
[3] Xiaohui Wang, Zhaosheng Feng, Lokenath Debnath and David Y. Gao (2008): The Korteweg-de Vries-Burgers equation and its approximate solution.- Int. J. Comput. Math., vol.85. pp.853-863. https://doi.org/10.1080/00207160701411152

[4] Caflisch R., Miksis M., Papanicolaou G. and Ting L. (1985): Wave propagation in bubbly liquids at finite volume fraction.- Journal of Fluid Mechanics, vol.160, pp.1-14. https://doi/org:10.1017/S0022112085003354

[5] Kudryashov N.A. and Sinelshchikov D.I. (2014): Extended models of non-linear waves in liquid with gas bubbles.International Journal of Non-Linear Mechanics, vol.63, pp.31-38. https://arxiv.org/ct?url=https\%3A\%2F\%2Fdx.doi.org\%2F10.1016\%2Fj.ijnonlinmec.2014.03.011\&v=1d590801

[6] Mahmood S. and Kwak H.Y. (2016): Pressure waves in bubbly liquids.- J Mech Sci Technol, vol.30, pp.3935-3943. https://doi.org/10.1007/s12206-016-0805-2

[7] Keller J.B. and Miksis M. (1980): Bubble oscillations of large amplitude.- J. Acoust. Soc. Am., vol.68, pp.628-633.

[8] Yuning Zhang, Zhongyu Guo, Yuhang Gao and Xiaoze Du (2018): Acoustic wave propagation in bubbly flow with gas, vapor or their mixtures.- Ultrasonics Sonochemistry, vol.40, Part B, pp.40-45. https://doi.org/10.1016/j.ultsonch.2017.03.048

[9] Seung S. and Kwak H.Y. (2017): Shock wave propagation in bubbly liquids at small gas volume fractions.- J Mech Sci Technol, vol.31, pp.1223-1231. https://doi.org/10.1007/s12206-017-0221-2

[10] Martins J.C. and Seleghim P. (2017): Propagation and attenuation of pressure waves in dispersed two-phase flows.J. Fluids Eng., vol.139, No.1, p.13. doi: https://doi.org/10.1115/1.4034370

[11] Gubaidullin D.A. and Fedorov Y.V. (2019): Acoustic waves in a liquid with gas bubbles covered by a viscoelastic shell.- Fluid Dyn, vol.54, pp.270-278. https://doi.org/10.1134/S0015462819010075

[12] Li Hongtao, Chen Ruoming, Li Xiaojun, Meng Yingfeng, Zhu Li and Zhao Jibin (2016): Investigation of pressure wave propagation and attenuation characteristics in wellbore gas-liquid two-phase flow.- Journal of Natural Gas Science and Engineering, vol.35. https://doi.org/10.1016/j.jngse.2016.09.020

[13] Yano T., Kanagawa T., Watanabe M. and Fujikawa S. (2013): Nonlinear Wave Propagation in Bubbly Liquids.- In: Bubble Dynamics and Shock Waves (C. Delale, Ed.), Shock Wave Science and Technology Reference Library, vol.8., Springer, Berlin, Heidelberg. https://doi.org/10.1007/978-3-642-34297-4_4

[14] Sitdikova L.F. and Gimaltdinov I.K. (2019): The problem spreading acoustic waves in a porous environment with air bubbles on pore walls.- In: International Scientific Conference Energy Management of Municipal Facilities and Sustainable Energy Technologies, 10-13 December 2019, Voronezh, Russian Federation, Journal of Physics: Conference Series, vol.1614. doi:10.1088/1742-6596/1614/1/012088

[15] Cerepi Adrian (2004): Streaming potential in two-phase flow condition.- Geophysical Research Letters, vol.31, No.11. doi.org/10.1029/2004GL020140

[16] Panahov G.M. and Museibli P.T (2017): The study of internal exposure on the fluid hydrodynamics.- Transactions of NAS of Azerbaijan: Issue Mechanics, vol.37, No.7, pp.66-71.

[17] Gallyamov A.K., Shammazov A.M., Tagirov R.Sh. and Fattakhov M.M. (1983): Investigation of the influence of gas on the accuracy of turbine flowmeters (in Russian).- Oil Industry, vol.4, pp.47-49

[18] Nigmatulin R.I. (1978): Fundamentals of Mechanics of Heterogeneous Media.- Moscow: Nauka.

[19] Nakoryakov V.E., Sobolev V.V. and Shreiber I.R. (1972): Longwave perturbations in a gas-liquid mixture.- Fluid Dyn, vol.7, pp.763768. https://doi.org/10.1007/BF01205753

[20] Kutateladze S.S. and Styrikovich M.A. (1976): Hydrodynamics Of Gas-Liquid Systems.- Moscow: Energia.

[21] Karpman V.I. (1973): Nonlinear Waves in Dispersive Media.- Moscow: Nauka.

[22] Whitham J. (1977): Linear and Nonlinear Waves.- Moscow: Mir, p.622.

Received: April 22, 2021

Revised: July 16, 2021 